\title{
Chow motives versus noncommutative motives
}

\author{
Gonçalo Tabuada*
}

\begin{abstract}
In this article we formalize and enhance Kontsevich's beautiful insight that Chow motives can be embedded into noncommutative ones after factoring out by the action of the Tate object. We illustrate the potential of this result by developing three of its manyfold applications: (1) the notions of Schur and Kimura finiteness admit an adequate extension to the realm of noncommutative motives; (2) Gillet-Soulé's motivic measure admits an extension to the Grothendieck ring of noncommutative motives; (3) certain motivic zeta functions admit an intrinsic construction inside the category of noncommutative motives.
\end{abstract}

Mathematics Subject Classification (2010). 14G10, 19E15, 19 F27.

Keywords. Chow motives, noncommutative motives, Kimura and Schur finiteness, motivic measures, motivic zeta functions.

\section{Introduction}

In the early sixties Grothendieck envisioned the existence of a "universal" cohomology theory of algebraic varieties. Among several conjectures and developments, a contravariant functor

$$
M: \mathrm{SmProj}^{\mathrm{op}} \rightarrow \mathrm{Chow}_{\mathbb{Q}}
$$

from smooth projective varieties (over a base field) towards a certain category of Chow motives was constructed; see $\$ 5.1$ for details. Intuitively, Chow $\mathbb{Q}$ encodes all the geometric/arithmetic information about smooth projective varieties and acts as a gateway between algebraic geometry and the assortment of numerous cohomology theories (de Rham, $l$-adic, crystalline, and others); for details consult the monograph [26].

During the last two decades Bondal, Drinfeld, Kaledin, Kapranov, Kontsevich, Van den Bergh, and others, have been promoting a broad noncommutative geometry program in which geometry is performed directly on triangulated categories and its differential graded enhancements; see [9], [11], [19], [20], [27], [34], [33], [36], [35]. One of the beauties of this program is its broadness. It encompasses several research fields such as algebraic geometry, representation theory of quivers, sympletic geometry, and even mathematical physics, making it a cornerstone of modern mathematics. In analogy with the commutative world, a central problem is the development of a

\footnotetext{
*The author was supported by the Portuguese Foundation for Science and Technology through the grants PTDC/MAT/098317/2008, SFRH/BSAB/1116/2011 and PEst-OE/MAT/UI0297/2011 (CMA).
} 
theory of noncommutative motives. By adapting the classical notions of smoothness and properness to the noncommutative world, Kontsevich introduced recently a category KMM of noncommutative motives; see $\$ 6.1$ for details. The following question is therefore of major importance.

Question. How to bridge the gap between Grothendieck's category of Chow motives and Kontsevich's category of noncommutative motives?

This question was settled by Kontsevich in [35], §4.1.3. The purpose of this article is to formalize and enhance his beautiful insight while illustrating its power through three applications.

Recall from $\$ 5.1$ that the category Chow $\mathbb{Q}_{\mathbb{Q}}$ is $\mathbb{Q}$-linear, additive and symmetric monoidal. Moreover, it is endowed with an important $\otimes$-invertible object, the Tate motive $\mathbb{Q}(1)$. The functor $-\otimes \mathbb{Q}(1)$ is an automorphism of Chow $\mathbb{Q}$ and so we can consider the associated orbit category Chow $\mathbb{Q} /-\otimes \mathbb{Q}(1)$; consult $\$ 7$ for the precise construction. Informally speaking, Chow motives which differ from a Tate twist become equal in the orbit category.

In the noncommutative world we have the category Hmo of dg categories up to derived Morita equivalence and a universal functor $U:$ Hmo $\rightarrow$ Mot, with values in a triangulated category, that preserves filtered homotopy colimits and sends exact sequences to distinguished triangles; see $\S 6$ for details. Recall that a dg category $\mathcal{A}$ is smooth and proper in the sense of Kontsevich if its complexes of morphisms are perfect and $\mathcal{A}$ is perfect as a bimodule over itself. As explained in $\S 6.1$, Kontsevich's category KMM of noncommutative motives can be identified with the smallest thick triangulated subcategory of Mot spanned by the objects $U(\mathcal{A})$, with $\mathcal{A}$ a smooth and proper dg category. By first taking rational coefficients $(-)_{\mathbb{Q}}$ and then passing to the idempotent completion $(-)^{\natural}$ we obtain in particular a natural inclusion $\mathrm{KMM}_{\mathbb{Q}}^{\natural} \subset$ $\operatorname{Mot}_{\mathbb{Q}}^{\natural}$; see $\S 6.2-6.3$.

The gap between algebraic varieties and dg categories can be bridged by associating to every smooth projective variety $X$ its dg category $\mathscr{D}_{\text {perf }}^{\mathrm{dg}}(X)$ of perfect complexes of $\mathcal{O}_{X}$-modules; see Lunts-Orlov [38] or [16], Example 4.5.

Theorem 1.1 (Kontsevich). There exists a fully faithful, $\mathbb{Q}$-linear, additive, and symmetric monoidal functor $R$ making the diagram

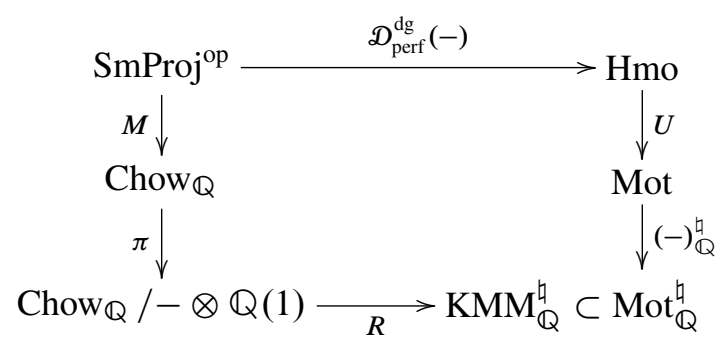

commutative up to a natural isomorphism. 
Intuitively speaking, Theorem 1.1 formalizes the conceptual idea that the commutative world can be embedded into the noncommutative world after factoring out by the action of the Tate object. In the next three sections we will illustrate the potential of this result by describing some of its manyfold applications.

\section{Schur and Kimura finiteness}

By construction, the category $\mathrm{KMM}_{\mathbb{Q}}^{\natural}$ is $\mathbb{Q}$-linear, idempotent complete and moreover symmetric monoidal. Hence, given a partition $\lambda$ of a natural number $n$, we can consider the associated Schur functor $\mathbf{S}_{\lambda}: \mathrm{KMM}_{\mathbb{Q}}^{\natural} \rightarrow \mathrm{KMM}_{\mathbb{Q}}^{\natural}$ which sends a noncommutative motive $N$ to the direct summand of $N^{\otimes n}$ determined by $\lambda$; see Deligne's foundational work [18]. We say that $N$ is Schur finite if it is annihilated by some Schur functor. When $\lambda=(n)$, resp. $\lambda=(1, \ldots, 1)$, the associated Schur functor $\operatorname{Sym}^{n}:=\mathbf{S}_{(n)}$, resp. Alt ${ }^{n}:=\mathbf{S}_{(1, \ldots, 1)}$, should be considered as the motivic analogue of the usual $n^{\text {th }}$ symmetric, resp. wedge, product functor of $\mathbb{Q}$-vector spaces. We say that $N$ is evenly, resp. oddly, finite dimensional if $\operatorname{Alt}^{n}(N)$, resp. $\operatorname{Sym}^{n}(N)$, vanishes for some $n$. Finally, $N$ is Kimura finite if it admits a direct sum decomposition $N \simeq N_{+} \oplus N_{-}$into a evenly $N_{+}$and oddly $N_{-}$finite dimensional object. Note that Kimura finiteness implies Schur finiteness.

In the commutative world, the aforementioned general finiteness notions were extensively studied by André-Kahn, Guletskii, Guletskii-Pedrini, Kimura, Mazza, and others; see [1], [3], [24], [25], [32], [40]. For instance, Guletskii and Pedrini proved that given a smooth projective surface $X$ (over a field of characteristic zero) with $p_{g}(X)=0$, the Chow motive $M(X)$ is Kimura finite if and only if Bloch's conjecture on the Albanese kernel for $X$ holds.

Theorem 1.1 establishes a precise relationship between the finiteness conditions on the commutative and noncommutative worlds. For simplicity, let us write NC for the composed functor $U\left(D_{\text {perf }}^{\mathrm{dg}}(-)\right)_{\mathbb{Q}}^{\natural}: \operatorname{SmProj}^{\text {op }} \rightarrow \mathrm{KMM}_{\mathbb{Q}}^{\natural}$.

Theorem 2.1. Let $X$ be a smooth projective variety.

(i) If $M(X)$ is Schur finite, resp. Kimura finite, in $\mathrm{Chow}_{\mathbb{Q}}$, then $\mathrm{NC}(X)$ is Schur finite, resp. Kimura finite, in $\mathrm{KMM}_{\mathbb{Q}}^{\natural}$.

(ii) The converse for Schur finiteness holds, i.e., if $\mathrm{NC}(X)$ is Schur finite in $\mathrm{KMM}_{\mathbb{Q}}^{\natural}$, then $M(X)$ is Schur finite in Chow $_{\mathbb{Q}}$.

Informally speaking, Theorem 2.1 shows that Schur finiteness is a notion which is insensitive to commutativity. A potential application of this fact is the use of noncommutative methods in order to prove Schur finiteness for certain Chow motives. In what concerns Kimura finiteness, recall from [1] that all the Chow motives of the form $M(X)$, with $X$ an abelian variety, are Kimura finite. Using Theorem 2.1 and 
the stability of Kimura finiteness under several constructions (see [24], [40]), we then obtain a large class of examples of Kimura finite noncommutative motives.

\section{Motivic measures}

Let $k$ be a field and $\operatorname{Var}_{k}$ the category of algebraic $k$-varieties. Recall that the Grothendieck ring $K_{0} \operatorname{Var}(k)$ of algebraic $k$-varieties is the abelian group generated by the isomorphism classes of objects in $\operatorname{Var}(k)$ modulo the scissor congruence relations $[X]=[Z]+[X \backslash Z]$, where $Z$ is a closed subvariety of $X$. Multiplication is given by the fiber product over $\operatorname{spec}(k)$. Although very important, the structure of this ring remains rather mysterious. In order to capture some of its flavor several motivic measures, i.e., ring homomorphisms $\mu: K_{0} \operatorname{Var}(k) \rightarrow A$ towards commutative rings, have been built; see [37]. For instance, Gillet-Soulé [22] constructed for any perfect field $k$ (of arbitrary characteristic) an interesting motivic measure $\mu_{\mathrm{GS}}: K_{0} \operatorname{Var}(k) \rightarrow$ $K_{0}\left(\mathrm{Chow}_{\mathbb{Q}}\right)$ with values in the Grothendieck ring of Chow motives. By definition, it sends the class $[X]$ of a smooth projective variety $X$ to the class $[M(X)]$ of the naturally associated Chow motive $M(X)$.

Recall that by construction $\mathrm{KMM}_{\mathbb{Q}}^{\natural}$ is a $\otimes$-triangulated category. Hence, we can consider its Grothendieck ring ${ }^{1} K_{0}\left(\mathrm{KMM}_{\mathbb{Q}}^{\natural}\right)$ defined in the standard way. Theorem 1.1 enables then the extension of Gillet-Soulé's motivic measure to the noncommutative world.

Theorem 3.1. Let $k$ be a perfect field (of arbitrary characteristic). Then the assignment $X \mapsto[\mathrm{NC}(X)] \in K_{0}\left(\mathrm{KMM}_{\mathbb{Q}}^{\natural}\right)$, where $X$ is a smooth projective $k$-variety, gives rise to a well-defined motivic measure

$$
\mu_{\mathrm{NC}}: K_{0} \operatorname{Var}(k) \rightarrow K_{0}\left(\mathrm{KMM}_{\mathbb{Q}}^{\natural}\right) .
$$

Remark 3.2. Making use of Bittner-Looijenga's [7], [37] presentation of $K_{0} \operatorname{Var}(k)$ in the case of a field $k$ of characteristic zero, Bondal-Larsen-Lunts constructed in [10] a motivic measure $\mu_{\mathrm{BLL}}$ with values in a certain ring $\Gamma(k)$ of pre-triangulated dg categories. Using arguments similar to those of [44], Prop. 7.3, it can be shown that in this particular case the motivic measure $\mu_{\mathrm{NC}}$ factors through $\mu_{\mathrm{BLL}}$ via a natural ring homomorphism

$$
\Gamma(k) \rightarrow K_{0}\left(\mathrm{KMM}_{\mathbb{Q}}^{\natural}\right) .
$$

Intuitively speaking, $\mu_{\mathrm{NC}}$ measures the information concerning algebraic varieties (modulo the scissor congruence relations) which can be recovered from their derived categories of perfect complexes.

\footnotetext{
${ }^{1}$ A precise relationship between $K_{0}(\mathrm{KMM})$ (and hence between $K_{0}\left(\mathrm{KMM}_{\mathbb{Q}}^{\natural}\right)$ ) and Toën's secondary $\mathrm{K}$-theory is described in [16], §8.4.
} 
Example 3.4. Assume that $k=\mathbb{C}$. Then the assignment

$$
X \mapsto \chi_{\mathrm{c}}(X):=\sum_{i \geq 0}(-1)^{i} \operatorname{dim} H^{i}\left(X, \Omega_{X}\right),
$$

for every smooth projective $\mathbb{C}$-variety $X$, gives rise to a well-defined $\mathbb{Z}$-valued motivic measure $\mu_{\chi_{c}}$; see [2]. Recall from [16], §8.4, that Hochschild homology HH gives rise to a $\otimes$-triangulated functor $\mathrm{KMM} \rightarrow \mathscr{D}_{\mathrm{c}}(\mathbb{C})$. Since $\mathscr{D}_{\mathrm{c}}(\mathbb{C})$ is idempotent complete and $\mathbb{Q}$-linear, this functor extends uniquely to a $\otimes$-triangulated functor $\overline{\mathrm{HH}}: \mathrm{KMM}_{\mathbb{Q}} \rightarrow \mathscr{D}_{\mathrm{c}}(\mathbb{C})$ and so it induces a ring homomorphism

$$
K_{0}\left(\mathrm{KMM}_{\mathbb{Q}}^{\natural}\right) \rightarrow K_{0}\left(\mathscr{D}_{\mathrm{c}}(\mathbb{C})\right)=\mathrm{K}_{0}(\mathbb{C})=\mathbb{Z} .
$$

The natural equalities

$$
[\overline{\mathrm{HH}}(\mathrm{NC}(X))]=\left[\mathrm{HH}\left(\mathscr{D}_{\text {perf }}^{\mathrm{dg}}(X)\right)\right]=[\mathrm{HH}(X)]=\sum_{i \geq 0}(-1)^{i} \operatorname{dim} H^{i}\left(X, \Omega_{X}\right),
$$

for every smooth projective $\mathbb{C}$-variety $X$, combined with Bittner-Looijenga's presentation of $K_{0} \operatorname{Var}(\mathbb{C})$ (see [7], [37]), allow us then to conclude that $\mu_{\chi_{\mathrm{c}}}$ factors through $\mu_{\mathrm{NC}}$ via the ring homomorphism (3.5). By combining this factorization with the one of Remark 3.2 we then obtain the illustrative diagram

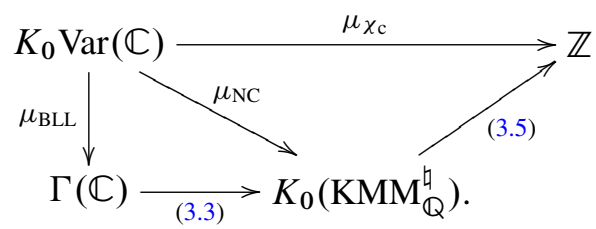

However, as the following non-example illustrates, the passage from the commutative to the noncommutative world forgets some geometric/arithmetic information.

Non-Example 3.6. Assume that $k=\mathbb{F}_{q}$, where $q=p^{n}$ with $p$ a prime number and $n$ a positive integer. Then the assignment $X \mapsto \# X\left(\mathbb{F}_{q}\right)$, for every smooth projective $\mathbb{F}_{q}$-variety $X$, gives rise to a well-defined $\mathbb{Z}$-valued motivic measure $\mu_{\sharp}$; see [2]. In contrast with $\mu_{\chi_{\mathrm{c}}}$, this motivic measure does not factor through $\mu_{\mathrm{NC}}$. Take for instance $X=\mathbb{P}^{1}$. Beilinson's [6] semi-orthogonal decomposition of $\mathscr{D}_{\text {perf }}^{\mathrm{dg}}\left(\mathbb{P}^{1}\right)$ into two copies of $\mathscr{D}_{\text {perf }}^{\mathrm{dg}}\left(\mathbb{F}_{q}\right)$ implies that $\mathrm{NC}\left(\mathbb{P}^{1}\right)=U\left(\mathbb{F}_{q}\right)_{\mathbb{Q}}^{\natural} \oplus U\left(\mathbb{F}_{q}\right)_{\mathbb{Q}}^{\natural}$. Hence, since $U\left(\mathbb{F}_{q}\right)_{\mathbb{Q}}^{\natural}$ is the unit of the $\otimes$-triangulated category $\mathrm{KMM}_{\mathbb{Q}}^{\natural}$, we conclude that $\left[\mathrm{NC}\left(\mathbb{P}^{1}\right)\right]=2$ in $K_{0}\left(\mathrm{KMM}_{\mathbb{Q}}^{\natural}\right)$. This implies that any motivic measure $\mu$ which factors through $\mu_{\mathrm{NC}}$ verifies the equality $\mu\left(\mathbb{P}^{1}\right)=2$, but this is clearly not the case for $\mu_{\#}$ since $\#\left(\mathbb{P}^{1}\right)=q+1$. Intuitively speaking, from the noncommutative motive $\mathrm{NC}(X)$ we can only recover the number of points of $X$ modulo $q-1$. 


\section{Motivic zeta functions}

Recall from Kapranov [28] that the motivic zeta function of a smooth algebraic $k$ variety $X$ with respect to a motivic measure $\mu$ is the following formal power series

$$
\zeta_{\mu}(X ; t):=\sum_{n=0}^{\infty} \mu\left(\left[S^{n}(X)\right]\right) t^{n} \in A[[t]],
$$

where $S^{n}(X)$ denotes the $n^{\text {th }}$ symmetric power of $X$. For instance, when $\mu$ is the motivic measure of the above Non-Example 3.6 we recover the classical Hasse-Weil zeta function.

On the other hand, recall that by construction $\mathrm{KMM}_{\mathbb{Q}}^{\natural}$ is an idempotent complete $\mathbb{Q}$-linear category. Hence, given any noncommutative motive $N$, we can construct its zeta function intrinsically inside $\mathrm{KMM}_{\mathbb{Q}}^{\natural}$ as follows:

$$
\zeta(N ; t):=\sum_{n=0}^{\infty}\left[\operatorname{Sym}^{n}(N)\right] t^{n} \in K_{0}\left(\mathrm{KMM}_{\mathbb{Q}}^{\natural}\right)[[t]] .
$$

Theorem 1.1 allows us then to compare this intrinsic construction with the motivic zeta function associated to the motivic measure $\mu_{\mathrm{NC}}$.

Theorem 4.1. Let $X$ a smooth projective $k$-variety. Then

$$
\zeta(\mathrm{NC}(X) ; t)=\zeta_{\mu_{\mathrm{NC}}}(X ; t) \in K_{0}\left(\mathrm{KMM}_{\mathbb{Q}}^{\natural}\right)[[t]] .
$$

As explained in [2], the motivic zeta function of a smooth projective $k$-variety $X$ with respect to the motivic measure of Example 3.4 is given by $(1-t)^{-\chi_{\mathrm{c}}(X)}$. Theorem 4.1 combined with Example 3.4 show us then that, in contrast with the Hasse-Weil zeta function, this information can be completely recovered solely from the intrinsic zeta function associated to the noncommutative motive $\mathrm{NC}(X)$.

\section{Some categories of motives}

In this section we recall, and adapt to our convenience, the construction of some categories of motives following Grothendieck, Manin, and Gillet-Soulé. These will be used in the proof of Theorem 1.1. In what follows, $k$ denotes a (fixed) base field.

5.1. Grothendieck's category of Chow motives (consult [43]). Given a smooth projective $k$-variety $X$ and an integer $d$, we will write $Z^{d}(X)$ for the $d$-codimensional cycle group of $X$ and $A^{d}(X):=\left(Z^{d}(X) \otimes_{\mathbb{Z}} \mathbb{Q}\right) /($ rational equivalence) for the $d$ codimensional Chow group with rational coefficients of $X$. If $Z$ is a cycle on $X$, we will denote by $[Z]$ its class in $A^{d}(X)$. Let $X$ and $Y$ be two smooth projective $k$-varieties, $X=\amalg_{i} X_{i}$ the decomposition of $X$ in its connected components, and 
$d_{i}$ the dimension of $X_{i}$. Then $\operatorname{Corr}^{r}(X, Y):=\bigoplus_{i} A^{d_{i}+r}\left(X_{i} \times Y\right)$ is called the space of correspondences of degree $r$ from $X$ to $Y$. Given $f \in \operatorname{Corr}^{r}(X, Y)$ and $g \in \operatorname{Corr}^{s}(Y, Z)$ their composition $g \circ f \in \operatorname{Corr}^{r+s}(X, Z)$ is defined by the classical formula

$$
g \circ f:=\left(\pi_{X Y}\right)_{*}\left(\pi_{X Y}^{*}(f) \cdot \pi_{Y Z}^{*}(g)\right) .
$$

The category Chow $\mathbb{Q}_{\mathbb{Q}}$ of Chow motives (with rational coefficients) is defined as follows: its objects are the triples $(X, p, m)$ where $X$ is a smooth projective $k$-variety, $m$ is an integer, and $p=p^{2} \in \operatorname{Corr}^{0}(X, X)$ is an idempotent endomorphism; its morphisms are given by

$$
\operatorname{Hom}_{C_{\text {how }}}((X, p, m),(Y, q, n)):=q \circ \operatorname{Corr}^{n-m}(X, Y) \circ p ;
$$

composition is induced by the above composition (5.1) of correspondences. By construction, the category $\mathrm{Chow}_{\mathbb{Q}}$ is $\mathbb{Q}$-linear, additive and pseudo-abelian (i.e., every idempotent endomorphism has a kernel). Moreover, it carries a symmetric monoidal structure defined on objects by the formula $(X, p, m) \otimes(Y, q, n):=$ $(X \times Y, p \otimes q, m+n)$. The unit object for this symmetric monoidal structure is the Chow motive $(\operatorname{spec}(k), \mathrm{id}, 0)$, where id $=[\Delta]$ is the class of the diagonal $\Delta$ in $\operatorname{Corr}^{0}(\operatorname{spec}(k), \operatorname{spec}(k))$. The Tate motive $(\operatorname{spec}(k)$, id, 1$)$ will be denoted by $\mathbb{Q}(1)$. Note that $\mathbb{Q}(1)$ is a $\otimes$-invertible object. Finally, we have a natural symmetric monoidal functor

$$
M: \text { SmProj }^{\mathrm{op}} \rightarrow \text { Chow }_{\mathbb{Q}}, \quad X \mapsto(X, \mathrm{id}, 0),
$$

which maps a morphism $f: X \rightarrow Y$ in SmProj to $\left[\Gamma_{f}^{t}\right]$, where $\Gamma_{f}^{t}$ is the transpose of the graph $\Gamma_{f}=\{(x, f(x)) \mid x \in X\} \subset X \times Y$ of $f$.

5.2. Manin's category of motives (consult [39]). The category $\mathrm{CHM}_{\mathbb{Q}}$ of Manin's motives (with rational coefficients) is defined as follows: its objects are the pairs $(X, p)$, where $X$ is a smooth projective $k$-variety and $p^{2}=p \in \operatorname{Corr}^{0}(X, X)$ is an idempotent endomorphism; its morphisms are given by

$$
\operatorname{Hom}_{\mathrm{CHM}_{\mathbb{Q}}}((X, p),(Y, q)):=q \circ \bigoplus_{j \in \mathbb{Z}} \operatorname{Corr}^{j}(X, Y) \circ p ;
$$

composition is induced by the above composition (5.1) of correspondences. Similarly to $\mathrm{Chow}_{\mathbb{Q}}$, the category $\mathrm{CHM}_{\mathbb{Q}}$ is $\mathbb{Q}$-linear, additive, pseudo-abelian, and symmetric monoidal. Moreover, we have a natural symmetric monoidal functor

$$
\mathrm{SmProj}^{\mathrm{op}} \rightarrow \mathrm{CHM}_{\mathbb{Q}}, \quad X \mapsto(X, \mathrm{id}) .
$$

5.3. Gillet-Soulé's category of motives (consult [22], [21]). The category $\mathrm{KM}_{\mathbb{Q}}$ of Gillet-Soulé's motives (with rational coefficients) is constructed in two steps. ${ }^{2}$ First,

\footnotetext{
${ }^{2}$ Gillet-Soulé did not consider the pseudo-abelianization procedure.
} 
consider the category $\underline{\mathrm{KM}}_{\mathbb{Q}}$ whose objects are the smooth projective $k$-varieties and whose morphisms are given by

$$
\operatorname{Hom}_{\underline{K M}}(X, Y):=K_{0}(X \times Y)_{\mathbb{Q}}=K_{0}(X \times Y) \otimes_{\mathbb{Z}} \mathbb{Q} .
$$

Composition is defined by composing the bilinear pairing

$$
\begin{aligned}
K_{0}(X \times Y)_{\mathbb{Q}} \times K_{0}(Y \times Z)_{\mathbb{Q}} & \rightarrow K_{0}(X \times Y \times Z)_{\mathbb{Q}}, \\
([\mathcal{F}],[\mathcal{E}]) & \mapsto \sum_{i \geq 0}(-1)^{i}\left[\operatorname{Tor}_{i}^{\mathcal{O}_{Y}}(\mathcal{F}, \mathscr{E})\right],
\end{aligned}
$$

with the direct image homomorphism $K_{0}(X \times Y \times Z)_{\mathbb{Q}} \rightarrow K_{0}(X \times Z)_{\mathbb{Q}}$ induced by the natural projection map. Gillet-Soulé used $G$-theory instead of K-theory. However, since we are working with smooth projective varieties over a field these theories agree; see Quillen [41], §7.1. Then take the pseudo-abelianization of $\underline{K M}_{\mathbb{Q}}$. The objects of the resulting category $\mathrm{KM}_{\mathbb{Q}}$ are the pairs $(X, p)$, where $X$ is a smooth projective $k$-variety and $p^{2}=p \in K_{0}(X \times X)_{\mathbb{Q}}$ is an idempotent endomorphism. Morphisms are given by

$$
\operatorname{Hom}_{\mathrm{KM}_{\mathbb{Q}}}((X, p),(Y, q)):=q \circ K_{0}(X \times Y)_{\mathbb{Q}} \circ p
$$

and composition is induced from the one on $\mathrm{KM}_{\mathbb{Q}}$. In particular, we have a natural fully faithful functor $\underline{K M}_{\mathbb{Q}} \rightarrow \mathrm{KM}_{\mathbb{Q}}$. By construction, $\mathrm{KM}_{\mathbb{Q}}$ is $\mathbb{Q}$-linear, additive and pseudo-abelian, Moreover, it carries a symmetric monoidal structure defined on objects by the formula $(X, p) \otimes(Y, q)=(X \times Y, p \otimes q)$. Finally, we have a natural symmetric monoidal functor

$$
\text { SmProj }^{\text {op }} \rightarrow \mathrm{KM}_{\mathbb{Q}}, \quad X \mapsto(X, \mathrm{id}),
$$

which maps a morphism $f: X \rightarrow Y$ in SmProj to $\left[\mathcal{O}_{\Gamma_{f}^{t}}\right] \in K_{0}(Y \times X)_{\mathbb{Q}}$.

\section{Noncommutative motives}

A differential graded (dg) category, over a fixed commutative base ring $k$, is a category enriched over cochain complexes of $k$-modules (morphisms sets are such complexes) in such a way that composition fulfills the Leibniz rule: $d(f \circ g)=(d f) \circ g+$ $(-1)^{\operatorname{deg}(f)} f \circ(d g)$; see Keller's ICM address [31]. As proved in [44], the category dgcat of dg categories carries a Quillen model structure whose weak equivalences are the derived Morita equivalences, i.e., the dg functors $F: \mathcal{A} \rightarrow \mathscr{B}$ which induce an equivalence $\mathscr{D}(\mathscr{A}) \stackrel{\sim}{\longrightarrow} \mathscr{D}(\mathscr{B})$ on derived categories; see [31], §4.6. The homotopy category obtained is denoted by Hmo.

All the classical (functorial) invariants, such as Hochschild homology, cyclic homology and its variants (periodic, negative, ...), algebraic K-theory, and even topological cyclic homology (see [46]), extend naturally from $k$-algebras to dg categories. 
In order to study all these classical invariants simultaneously, the notion of localizing invariant was introduced in [45]. This notion, that we now recall, makes use of the language of Grothendieck derivators, a formalism which allows us to state and prove precise universal properties; consult Appendix 9. Let $L: \mathrm{HO}($ dgcat $) \rightarrow \mathbb{D}$ be a morphism of derivators, from the derivator associated to the derived Morita model structure, towards a triangulated derivator $\mathbb{D}$. We say that $L$ is a localizing invariant if it preserves filtered homotopy colimits and sends Drinfeld's exact sequences of dg categories (see [19]) to distinguished triangles

$$
\mathcal{A} \rightarrow \mathscr{B} \rightarrow \mathcal{C} \mapsto L(\mathcal{A}) \rightarrow L(\mathscr{B}) \rightarrow L(\mathcal{C}) \rightarrow \Sigma(L(\mathcal{A}))
$$

in the base category $\mathbb{D}(e)$ of $\mathbb{D}$. Thanks to the work of Blumberg-Mandell, Keller, Schlichting, and Thomason-Trobaugh (see [8], [30], [29], [42], [47]), all the mentioned invariants satisfy localization ${ }^{3}$ and so give rise to localizing invariants. In [45], the universal localizing invariant (with values in a strong triangulated derivator) was constructed

$$
\text { U: } \mathrm{HO}(\text { dgcat }) \rightarrow \text { Mot } .
$$

Given any strong triangulated derivator $\mathbb{D}$, we have an induced equivalence of categories

$$
u^{*}: \underline{\operatorname{Hom}}_{!}(\mathcal{M} o t, \mathbb{D}) \stackrel{\sim}{\longrightarrow} \underline{\operatorname{Hom}}_{\mathrm{loc}}(\mathrm{HO}(\text { dgcat }), \mathbb{D}),
$$

where the right hand-side denotes the category of localizing invariants. Because of this universality property, which is a reminiscence of motives, $\mathcal{U}$ is called the universal localizing invariant and $\mathcal{M}$ ot the localizing motivator.

Notation. In order to simplify the exposition, the base category $\mathcal{M} o t(e)$ of the localizing motivator will be denoted by Mot and called the triangulated category of noncommutative motives (over $k$ ). The evaluation of the universal localizing invariant $U$ at the base category $e$ will be denoted by

$$
U: \mathrm{Hmo}=\mathrm{HO}(\text { dgcat })(e) \stackrel{U(e)}{\longrightarrow} \text { Mot. }
$$

Remark 6.3. The localizing motivator $\mathcal{M} o t$ (and hence the triangulated category Mot) is characterized by the above universal property (6.1). A Quillen model for $\mathcal{M} o t$ (and hence for Mot), given in terms of a Bousfield localization of presheaves of (symmetric) spectra, can be found in [45], §11.

The tensor product of $k$-algebras extends naturally to dg categories, giving rise to a symmetric monoidal structure on $\mathrm{HO}$ (dgcat). The corresponding unit is the dg category $\underline{k}$ with a single object and with the base ring $k$ as the dg algebra of endomorphisms. Using Day's (derived) convolution product, this monoidal structure on $\mathrm{HO}$ (dgcat) was extended (in a universal way) to Mot. The universal localizing

\footnotetext{
${ }^{3}$ In the case of algebraic K-theory we consider its non-connective version.
} 
invariant $U$ becomes symmetric monoidal and the equivalence (6.1) admits an appropriate symmetric monoidal enhancement; see [16]. In particular, the category Mot is $\otimes$-triangulated and the functor (6.2) is symmetric monoidal.

6.1. Kontsevich's category. A dg category $\mathcal{A}$ is called proper if for each ordered pair of objects $(x, y)$ in $\mathcal{A}$ the cochain complex of $k$-modules $\mathcal{A}(x, y)$ is perfect, and smooth if it is perfect as a bimodule over itself. As explained in [34], Kontsevich's construction of the category KMM of noncommutative motives decomposes in three steps:

(i) First consider the category KPM (enriched over spectra) whose objects are the smooth and proper dg categories and whose morphisms from $\mathcal{A}$ to $\mathscr{B}$ are given by the non-connective $\mathrm{K}$-theory spectrum $K\left(\mathcal{A}^{\mathrm{op}} \otimes \mathscr{B}\right)$. Composition corresponds to the (derived) tensor product of bimodules.

(ii) Then take the formal triangulated envelope of KPM. Objects in this new category are formal finite extensions of formal shifts of objects in KPM.

(iii) Finally, add formal direct summands for idempotent endomorphisms and pass to the underlying homotopy category. The resulting category KMM is in particular triangulated and its morphisms are given in terms of $\mathrm{K}$-theory groups.

In [16], Prop. 8.5, Kontsevich's construction is characterized in a simple and elegant way: KMM identifies with the thick triangulated subcategory of Mot spanned by the objects $U(\mathcal{A})$, with $\mathcal{A}$ a smooth and proper dg category. In particular, KMM $\subset$ Mot. For smooth and proper dg categories $\mathcal{A}$ and $\mathscr{B}$, we have

$$
\operatorname{Hom}_{\mathrm{KMM}}(U(\mathcal{A}), U(\mathscr{B})) \simeq K_{0}\left(\mathscr{A}^{\text {op }} \otimes \mathscr{B}\right)
$$

with composition given by the (derived) tensor product of bimodules. Finally, note that since smooth and proper dg categories are stable under tensor product, KMM is moreover a $\otimes$-triangulated subcategory of Mot.

6.2. Rational coefficients. In this subsection we assume that $k$ is a field. Let $\mathrm{KMM}_{\mathbb{Q}}$, resp. Mot $\mathbb{Q}_{\mathbb{Q}}$, be the category obtained form KMM, resp. from Mot, by tensoring each abelian group of morphisms with $\mathbb{Q}$. By construction, we have natural functors

$$
(-)_{\mathbb{Q}}: \mathrm{KMM} \rightarrow \mathrm{KMM}_{\mathbb{Q}}, \quad(-)_{\mathbb{Q}}: \operatorname{Mot} \rightarrow \operatorname{Mot}_{\mathbb{Q}} .
$$

Lemma 6.6. The categories $\mathrm{KMM}_{\mathbb{Q}}$ and $\mathrm{Mot}_{\mathbb{Q}}$ inherit from $\mathrm{KMM}$ and Mot, respectively, a canonical $\otimes$-triangulated structure making the natural functors (6.5) $\otimes$-triangulated.

Proof. Since both cases are similar we focus on the category $\operatorname{Mot}_{\mathbb{Q}}$. The dg category $\underline{k}$ is smooth and proper and so the above isomorphism (6.4) restricts to

$$
\operatorname{Hom}_{M o t}(U(\underline{k}), U(\underline{k})) \simeq K_{0}(k) .
$$


Since by hypothesis $k$ is a field, we conclude that the endomorphism ring of the unit $U(\underline{k})$ of the $\otimes$-triangulated category Mot is $K_{0}(k)=\mathbb{Z}$. Hence, by applying [4], Thm. 3.6, to the multiplicative set $S:=\mathbb{Z} \backslash\{0\} \subset K_{0}(k)$ of the endomorphism ring of $U(\underline{k})$ we obtain a canonical $\otimes$-triangulated structure on $\operatorname{Mot}_{\mathbb{Q}}$ making the functor $(-)_{\mathbb{Q}} \otimes$-triangulated.

6.3. Idempotent completion. The $\otimes$-triangulated categories $K M M_{\mathbb{Q}}$ and $\operatorname{Mot}_{\mathbb{Q}}$ of the previous section are not idempotent complete. Thanks to Balmer-Schlichting [5], their idempotent completions $\mathrm{KMM}_{\mathbb{Q}}^{\natural}$ and $\operatorname{Mot}_{\mathbb{Q}}^{\natural}$ carry a canonical triangulated structure. By construction they carry also a canonical $\otimes$-structure. Hence, we obtain natural $\otimes$-triangulated functors

$$
(-)^{\natural}: \mathrm{KMM}_{\mathbb{Q}} \rightarrow \mathrm{KMM}_{\mathbb{Q}}^{\natural}, \quad(-)^{\natural}: \operatorname{Mot}_{\mathbb{Q}} \rightarrow \operatorname{Mot}_{\mathbb{Q}}^{\natural} .
$$

\section{Orbit categories}

Let $\mathcal{C}$ be an additive category and $F: \mathcal{C} \rightarrow \mathcal{C}$ an automorphism (a standard construction allow us to replace an autoequivalence by an automorphism). By definition, the orbit category $\mathcal{E} / F$ has the same objects as $\mathcal{C}$ and morphisms

$$
\operatorname{Hom}_{\mathcal{C} / F}(X, Y):=\bigoplus_{j \in \mathbb{Z}} \operatorname{Hom}_{\mathcal{C}}\left(X, F^{j} Y\right) \text {. }
$$

Composition is induced by the one on $\mathcal{C}$. More precisely, given objects $X, Y, Z$ and morphisms

$$
\underline{f}=\left\{f_{j}\right\}_{j \in \mathbb{Z}} \in \bigoplus_{j \in \mathbb{Z}} \operatorname{Hom}_{e}\left(X, F^{j} Y\right), \quad \underline{g}=\left\{g_{l}\right\}_{l \in \mathbb{Z}} \in \bigoplus_{l \in \mathbb{Z}} \operatorname{Hom}_{\bigodot}\left(Y, F^{l} Z\right),
$$

the $i^{\text {th }}$-component of the composition $\underline{g} \circ \underline{f}$ is given by the finite sum

$$
\sum_{j} F^{j}\left(g_{i-j}\right) \circ f_{j}
$$

The canonical projection functor

$$
\pi: \mathcal{C} \rightarrow \mathcal{C} / F, \quad X \mapsto X, \quad f \mapsto \underline{f}=\left\{f_{j}\right\}_{j \in \mathbb{Z}},
$$

where $f_{0}=f$ and $f_{j}=0$ for $j \neq 0$, is endowed with a natural isomorphism $\pi \circ F \stackrel{\Longrightarrow}{\Longrightarrow} \pi$ and is universal among all such functors. By construction, $\mathcal{C} / F$ is still an additive category and the projection is an additive functor.

Remark 7.2. Let $X$ be an object in $\mathcal{\ell}, m$ an integer, and $\underline{\mu}$ and $\underline{v}$ the following morphisms in $\mathcal{C} / F$

$$
\underline{\mu}: X \rightarrow F^{m} X \quad \underline{v}: F^{m} X \rightarrow X,
$$


where $\mu_{j}=\operatorname{id}_{X}$ for $j=-m$ and $\mu_{j}=0$ for $j \neq-m$, and $v_{j}=\operatorname{id}_{F^{m}}{ }_{X}$ for $j=m$ and $v_{j}=0$ for $j \neq m$. Then the above composition formula (7.1) allow us to conclude that $\mu$ and $\underline{v}$ are inverse of each other. As a consequence, $X$ is canonically isomorphic in the orbit category $\ell / F$ to all the objects of the form $F^{m} X, m \in \mathbb{Z}$.

Let us now suppose that $\mathcal{C}$ is moreover endowed with a symmetric monoidal structure such that the tensor product $-\otimes-$ is bi-additive.

Lemma 7.3. If the automorphism $F$ is of the form $-\otimes \mathcal{O}$, with $\mathcal{O}$ a $\otimes$-invertible object in $\mathcal{C}$, then the orbit category $\mathcal{C} /-\otimes \mathcal{O}$ inherits a natural symmetric monoidal structure making the projection functor $\pi$ symmetric monoidal.

Proof. Let us start by defining the tensor product on $\mathcal{C} /-\otimes \mathcal{O}$. On objects it is the same as the one on $\mathcal{C}$. On morphisms let

$$
\begin{aligned}
\bigoplus_{j \in \mathbb{Z}} \operatorname{Hom}_{\mathcal{C}}\left(X, Y \otimes \mathcal{O}^{j}\right) \times \bigoplus_{j \in \mathbb{Z}} \operatorname{Hom}_{\mathcal{C}}\left(Z, W \otimes \mathcal{O}^{j}\right) \\
\rightarrow \bigoplus_{j \in \mathbb{Z}} \operatorname{Hom}_{\mathcal{C}}\left(X \otimes Z,(Y \otimes W) \otimes \mathcal{O}^{j}\right)
\end{aligned}
$$

be the unique bilinear morphism that sends the homogeneous maps $f: X \rightarrow Y \otimes \mathcal{O}^{r}$ and $g: Z \rightarrow W \otimes \mathcal{O}^{s}$ to the homogeneous map

$$
X \otimes Z \stackrel{f \otimes g}{\longrightarrow} Y \otimes \mathcal{O}^{r} \otimes W \otimes \mathcal{O}^{s} \stackrel{\tau}{\rightarrow}(Y \otimes W) \otimes \mathcal{O}^{(r+s)},
$$

where $\tau$ is the commutativity isomorphism constraint. The associativity and commutativity isomorphism constraints are obtain from the corresponding ones of $\mathcal{C}$ by applying the projection functor $\pi$. A routine verification shows that these definitions endow the orbit category $\mathcal{C} /-\otimes \mathcal{O}$ with a symmetric monoidal structure making the projection functor $\pi$ symmetric monoidal.

\section{Proof of Theorem 1.1}

Proof. The bulk of the proof consists on constructing functors $\theta_{1}, \theta_{2}$ and $\theta_{3}$ making the diagram

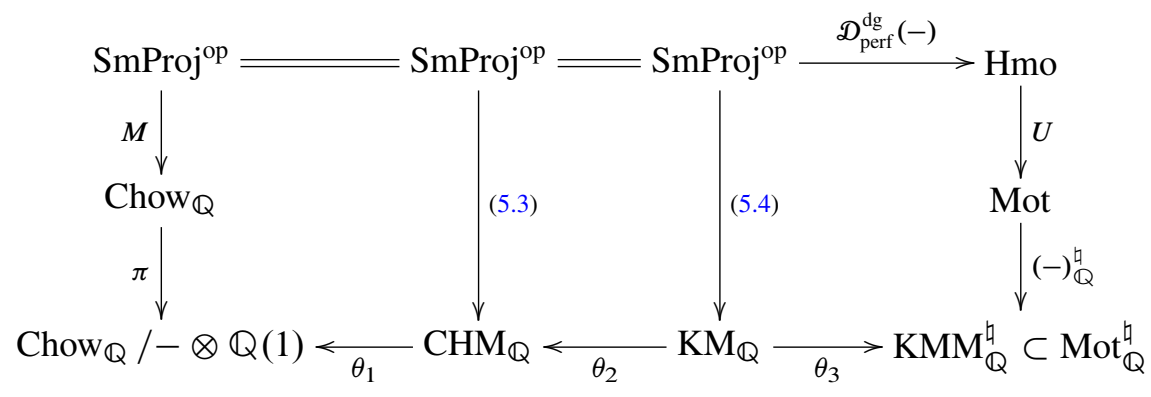


commutative up to a natural isomorphism. Let us start with the functor $\theta_{1}$. Given Chow motives $(X, p, m)$ and $(Y, q, n)$ the description of the orbit category given in the previous section show us that

$$
\operatorname{Hom}_{\mathrm{Chow}_{\mathbb{Q}} /-\otimes \mathbb{Q}(1)}((X, p, m),(Y, q, n))=q \circ \bigoplus_{j \in \mathbb{Z}} \operatorname{Corr}^{(n+j)-m}(X, Y) \circ p .
$$

Hence we define $\theta_{1}$ to be the functor that maps $(X, p)$ to $(X, p, 0)$ and which is the identity on morphisms. By construction, it is clearly fully faithful. In order to show that it is also essentially surjective, we need to prove that every object $(X, p, m)$ in Chow $_{\mathbb{Q}} /-\otimes \mathbb{Q}(1)$ is isomorphic to an object of the form $(X, p, 0)$. Since $(X, p, m)=(X, p, 0) \otimes \mathbb{Q}(1)^{m}$, this follows from Remark 7.2 (applied to the category $\mathcal{C}=$ Chow $\mathbb{Q}_{\text {and }}$ to the functor $F=-\otimes \mathbb{Q}(1)$ ). As a consequence, we conclude that the functor $\theta_{1}$ is an equivalence of categories. Note that it makes the left rectangle in the above diagram commute up to a natural isomorphism. Moreover, it is $\mathbb{Q}$-linear, additive and symmetric monoidal.

Let us now focus our attention on the functor $\theta_{2}$. Similarly to $K M_{\mathbb{Q}}$, the category $\mathrm{CHM}_{\mathbb{Q}}$ is obtained as the pseudo-abelianization of a category $\underline{\mathrm{CHM}}_{\mathbb{Q}}$ whose objects are the smooth projective $k$-varieties. Hence, we define $\theta_{2}$ to be the pseudoabelianization of the auxiliary functor $\widetilde{\theta_{2}}: \underline{\mathrm{KM}}_{\mathbb{Q}} \rightarrow \underline{\mathrm{CHM}}_{\mathbb{Q}}, X \mapsto X$, defined as follows

$$
K_{0}(X \times Y)_{\mathbb{Q}} \rightarrow \bigoplus_{j \in \mathbb{Z}} \operatorname{Corr}^{j}(X, Y), \quad \alpha \mapsto \operatorname{ch}(\alpha) \cdot \pi_{Y}^{*}(\operatorname{Td}(Y)),
$$

where ch denotes the Chern character and Td the Todd genus. Note that the Grothendieck-Riemann-Roch theorem guarantees that this functor $\widetilde{\theta_{2}}$ is well defined; see [22], p. 39. Moreover, it is fully faithful and induces a bijection on objects. Hence, the associated functor $\theta_{2}$ is an equivalence of categories. Finally, the GrothendieckRiemann-Roch theorem and the fact that the functors (5.3) and (5.4) factor through $\underline{\mathrm{CHM}}_{\mathbb{Q}}$ and $\underline{\mathrm{KM}}_{\mathbb{Q}}$, respectively, allow us to conclude that the middle rectangle of the above diagram is commutative up to a natural isomorphism. Note also that by construction $\theta_{2}$ is $\mathbb{Q}$-linear, additive and symmetric monoidal.

Let us now consider the functor $\theta_{3}$. Recall from $\S 5.3$ and $\S 6.3$ that we have a pseudo-abelianization functor $\underline{\mathrm{KM}}_{\mathbb{Q}} \rightarrow \mathrm{KM} \mathbb{Q}_{\mathbb{Q}}$ and an idempotent completion functor $\mathrm{KMM}_{\mathbb{Q}} \rightarrow \mathrm{KMM}_{\mathbb{Q}}^{\natural}$. We will then construct an auxiliary functor $\widetilde{\theta_{3}}: \underline{\mathrm{KM}}_{\mathbb{Q}} \rightarrow$ $\mathrm{KMM}_{\mathbb{Q}}$ and define $\theta_{3}$ as the canonical extension of the composed functor

$$
\underline{\mathrm{KM}}_{\mathbb{Q}} \stackrel{\widetilde{\theta_{3}}}{\rightarrow} \mathrm{KMM}_{\mathbb{Q}} \stackrel{(-)^{\natural}}{\longrightarrow} \mathrm{KMM}_{\mathbb{Q}}^{\natural}
$$

to $\mathrm{KM}_{\mathbb{Q}}$. Given smooth projective $k$-varieties $X$ and $Y$ we have the following natural 
isomorphisms:

$$
\begin{aligned}
\operatorname{Hom}_{\mathrm{KMM}}\left(U\left(\mathscr{D}_{\text {perf }}^{\mathrm{dg}}(X)\right)_{\mathbb{Q}}, U\left(\mathscr{D}_{\text {perf }}^{\mathrm{dg}}(Y)\right)_{\mathbb{Q}}\right) & =K_{0}\left(\left(\mathscr{D}_{\text {perf }}^{\mathrm{dg}}(X)\right)^{\mathrm{op}} \otimes \mathscr{D}_{\text {perf }}^{\mathrm{dg}}(Y)\right)_{\mathbb{Q}} \\
& \simeq K_{0}\left(\mathscr{D}_{\text {perf }}^{\mathrm{dg}}(X) \otimes \mathcal{D}_{\text {perf }}^{\mathrm{dg}}(Y)\right)_{\mathbb{Q}} \\
& \simeq K_{0}\left(\mathscr{D}_{\text {perf }}^{\mathrm{dg}}(X \times Y)\right)_{\mathbb{Q}} \\
& \simeq K_{0}(X \times Y)_{\mathbb{Q}}
\end{aligned}
$$

Isomorphism (8.1) follows from the fact that the dg category $\mathscr{D}_{\text {perf }}^{\mathrm{dg}}(X)$ is self-dual, Isomorphism (8.2) from the natural isomorphism $\mathscr{D}_{\text {perf }}^{\mathrm{dg}}(X) \otimes \mathcal{D}_{\text {perf }}^{\mathrm{dg}}(Y) \simeq \mathscr{D}_{\text {perf }}^{\mathrm{dg}}(X \times Y)$ in Hmo, and Isomorphism (8.3) from the classical fact that the Grothendieck group of an algebraic variety can be recovered from its derived category of perfect complexes. Under these isomorphisms, the composition operation in $\mathrm{KMM}_{\mathbb{Q}}$ corresponds to the composition of the bilinear pairing

$$
\begin{aligned}
K_{0}(X \times Y)_{\mathbb{Q}} \times K_{0}(Y \times Z)_{\mathbb{Q}} & \rightarrow K_{0}(X \times Y \times Z)_{\mathbb{Q}}, \\
([\mathcal{F}],[\mathcal{E}]) & \mapsto \sum_{i \geq 0}(-1)^{i}\left[\operatorname{Tor}_{i}^{\mathcal{O}_{Y}}(\mathcal{F}, \mathcal{E})\right],
\end{aligned}
$$

with the direct image homomorphism $K_{0}(X \times Y \times Z)_{\mathbb{Q}} \rightarrow K_{0}(X \times Z)_{\mathbb{Q}}$ induced by the natural projection map. Hence, we define $\widetilde{\theta_{3}}$ as the functor that sends a smooth projective $k$-variety $X$ to $U\left(D_{\text {perf }}^{\mathrm{dg}}(X)_{\mathbb{Q}}\right)$ and which is the identity on morphisms (via the isomorphisms (8.1)-(8.3)). By construction, we obtain then a fully faithful functor $\theta_{3}$ which makes the right rectangle in the above diagram commute up to a natural isomorphism. Moreover, it is $\mathbb{Q}$-linear, additive and symmetric monoidal.

Finally, choose inverse functors $\theta_{1}^{-1}$ and $\theta_{2}^{-1}$ to $\theta_{1}$ and $\theta_{2}$ and define $R$ as the composition $\theta_{3} \circ \theta_{2}^{-1} \circ \theta_{1}^{-1}$. By construction, $R$ is fully faithful and makes the diagram (1.2) commute up to a natural isomorphism. The fact that it is $\mathbb{Q}$-linear and additive follows from the corresponding properties of the functors $\theta_{1}, \theta_{2}$ and $\theta_{3}$. The fact that it is moreover symmetric monoidal follows from the general Lemma 8.4 applied to the functors $\theta_{1}$ and $\theta_{2}$, and from the fact that $\theta_{3}$ is symmetric monoidal.

Lemma 8.4. Let $\left(\mathcal{C}, \otimes_{\mathcal{C}}, \mathbf{1}_{\mathcal{C}}\right)$ and $\left(\mathcal{C}, \otimes_{\mathfrak{D}}, \mathbf{1}_{\mathfrak{D}}\right)$ be two symmetric monoidal categories and $F: \mathcal{C} \stackrel{\sim}{\longrightarrow}$ a symmetric monoidal equivalence. Then every inverse functor $G: D \stackrel{\mathcal{C}}{\longrightarrow}$ of $F$ is naturally symmetric monoidal.

Proof. Let $G$ be an inverse functor of $F$. Then we have a natural adjunction

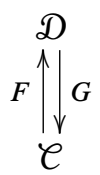


with $G$ right adjoint to $F$. The unit, resp. counit, of this adjunction will be denoted by $\eta$ : Id $\smile \cong G \circ F$ resp. by $\epsilon: F \circ G \stackrel{\Longrightarrow}{\Longrightarrow} \operatorname{Id}_{\mathscr{D}}$. Since $F$ is symmetric monoidal, we have natural isomorphisms

$$
v_{\mathbf{1}}^{F}: \mathbf{1}_{\mathfrak{D}} \stackrel{\sim}{\longrightarrow} F\left(\mathbf{1}_{\mathcal{C}}\right), \quad \mu_{c_{1}, c_{2}}^{F}: F\left(c_{1}\right) \otimes_{\mathbb{D}} F\left(c_{2}\right) \stackrel{\sim}{\longrightarrow} F\left(c_{1} \otimes_{\complement} c_{2}\right),
$$

which are coherently associative and unital (see diagrams 6.27-6.29 of [12]).

Now let $v_{1}^{G}: \mathbf{1}_{e} \rightarrow G\left(\mathbf{1}_{\mathfrak{D}}\right)$ be the morphism associated (under the above adjunction (8.5)) to the inverse $\left(v_{\mathbf{1}}^{F}\right)^{-1}$ of $v_{\mathbf{1}}^{F}$. Given objects $d_{1}, d_{2} \in \mathscr{D}$, let $\mu_{d_{1}, d_{2}}^{G}$ be the morphism associated (under the above adjunction (8.5)) to the following composed isomorphism

$$
F\left(G\left(d_{1}\right) \otimes_{e} G\left(d_{2}\right)\right) \stackrel{\left(\mu_{G\left(d_{1}\right), G\left(d_{2}\right)}^{F}\right)^{-1}}{\longrightarrow}(F \circ G)\left(d_{1}\right) \otimes_{\mathcal{D}}(F \circ G)\left(d_{2}\right)
$$

The functor $G$ endowed with the morphisms $\nu_{1}^{G}$ and $\mu_{d_{1}, d_{2}}^{G}$ is a lax symmetric monoidal functor. Note that since the above adjunction (8.5) is an equivalence of categories we have the following commutative diagrams:
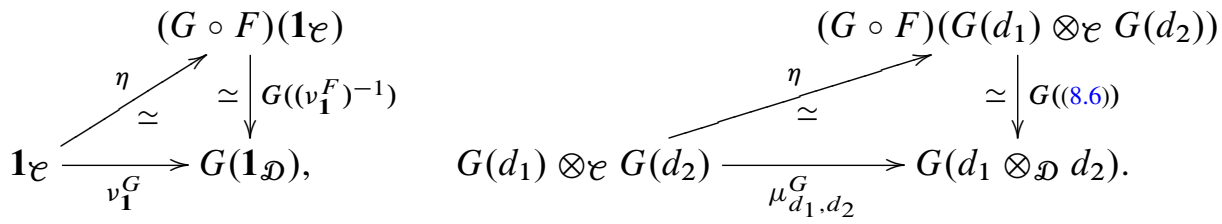

This allow us to conclude that the morphisms $v_{1}^{G}$ and $\mu_{d_{1}, d_{2}}^{G}$ are isomorphisms. As a consequence, we conclude that the functor $G$ is symmetric monoidal.

\section{Remaining proofs}

Theorem 2.1. Recall from [18] that Schur and Kimura finiteness is preserved by symmetric monoidal $\mathbb{Q}$-linear functors. Hence, item (i) follows from the commutativity of diagram (1.2) and from the fact that both functors $\pi$ and $R$ are $\mathbb{Q}$-linear and symmetric monoidal. Item (ii) follows from the faithfulness of $\pi$ and $R$.

Theorem 3.1. Since the functors $\pi$ and $R$ in diagram (1.2) are both additive and symmetric monoidal, their composite gives rise to a ring homomorphism

$$
K_{0}\left(\mathrm{Chow}_{\mathbb{Q}}\right) \rightarrow K_{0}\left(\mathrm{KMM}_{\mathbb{Q}}^{\natural}\right) .
$$

Recall from [22], Corollary 5.13, that Gillet-Soulé's motivic measure $\mu_{\mathrm{GS}}$ sends a smooth projective $k$-variety $X$ to $[M(X)]$. Hence, by composing (9.1) with $\mu_{\mathrm{GS}}$ we obtain a well-defined motivic measure $\mu_{\mathrm{NC}}$ sending a smooth projective $k$-variety $X$ to $[\mathrm{NC}(X)]$. 
Theorem 4.1. By definition of the formal power series $\zeta(\mathrm{NC}(X) ; t)$ and $\zeta_{\mu_{\mathrm{NC}}}(X ; t)$, it suffices to show the equality

$$
\left[\operatorname{Sym}^{n} \mathrm{NC}(X)\right]=\mu_{\mathrm{NC}}\left(\left[S^{n}(X)\right]\right) \in K_{0}\left(\mathrm{KMM}_{\mathbb{Q}}^{\natural}\right)
$$

for every natural number $n$. A similar equality

$$
\left[\operatorname{Sym}^{n} M(X)\right]=\mu_{\mathrm{GS}}\left(\left[S^{n}(X)\right]\right) \in K_{0}\left(\operatorname{Chow}_{\mathbb{Q}}\right)
$$

in the setting of Chow motives was proved by del Baño-Aznar in [17]. Since the functors $\pi$ and $R$ in diagram (1.2) are both symmetric monoidal and $\mathbb{Q}$-linear, their composition gives rise to a ring homomorphism

$$
\mathrm{K}_{0}\left(\mathrm{Chow}_{\mathbb{Q}}\right) \rightarrow K_{0}\left(\mathrm{KMM}_{\mathbb{Q}}^{\natural}\right)
$$

which maps $\left[\operatorname{Sym}^{n} M(X)\right]$ to $\left[\operatorname{Sym}^{n} \mathrm{NC}(X)\right]$. Hence, by applying this ring homomorphism (9.4) to the equalities (9.3) we obtain the searched equalities (9.2).

\section{Appendix. Grothendieck derivators}

The original reference for the theory of derivators is Grothendieck's original manuscript [23]. See also a short account on Cisinski-Neeman [14], §1. Derivators originate in the problem of higher homotopies in derived categories. For a triangulated category $\mathcal{T}$ and for $X$ a small category, it essentially never happens that the diagram category $\operatorname{Fun}(X, \mathcal{T})=\mathcal{T}^{X}$ remains triangulated; it already fails for the category of arrows in $\mathcal{T}$, that is, for $X=(\bullet \rightarrow \bullet)$.

Now, very often, our triangulated category $\mathcal{T}$ appears as the homotopy category $\mathcal{T}=\operatorname{Ho}(\mathcal{M})$ of some Quillen model $\mathcal{M}$. In this case, we can consider the category $\operatorname{Fun}(X, \mathcal{M})$ of diagrams in $\mathcal{M}$, whose homotopy category $\operatorname{Ho}(\operatorname{Fun}(X, \mathcal{M}))$ is often triangulated and provides a reasonable approximation for $\operatorname{Fun}(X, \mathcal{T})$. More importantly, one can let $X$ move. This nebula of categories $\operatorname{Ho}(\operatorname{Fun}(X, \mathcal{M}))$, indexed by small categories $X$, and the various functors and natural transformations between them is what Grothendieck formalized into the concept of derivator.

A derivator $\mathbb{D}$ consists of a strict contravariant 2-functor from the 2-category of small categories to the 2-category of all categories

$$
\mathbb{D}: \mathrm{Cat}^{\mathrm{op}} \rightarrow \mathrm{CAT}
$$

subject to certain conditions; consult [14], $\$ 1$, for details. The essential example to keep in mind is the derivator $\mathbb{D}=\mathrm{HO}(\mathcal{M})$ associated to a (cofibrantly generated) Quillen model category $\mathcal{M}$ and defined for every small category $X$ by

$$
\operatorname{HO}(\mathcal{M})(X)=\operatorname{Ho}\left(\operatorname{Fun}\left(X^{\mathrm{op}}, \mathcal{M}\right)\right)
$$


We denote by $e$ the 1-point category with one object and one identity morphism. Heuristically, the category $\mathbb{D}(e)$ is the basic "derived" category under consideration in the derivator $\mathbb{D}$. For instance, if $\mathbb{D}=\operatorname{HO}(\mathcal{M})$ then $\mathbb{D}(e)=\operatorname{Ho}(\mathcal{M})$. Let us now recall two slightly technical properties of derivators.

- A derivator $\mathbb{D}$ is called strong if for every finite free category $X$ and every small category $Y$, the natural functor $\mathbb{D}(X \times Y) \longrightarrow \operatorname{Fun}\left(X^{\mathrm{op}}, \mathbb{D}(Y)\right)$ is full and essentially surjective.

- A derivator $\mathbb{D}$ is called triangulated (or stable) if it is pointed and if every global commutative square in $\mathbb{D}$ is cartesian exactly when it is cocartesian; see [14], Def. 1.15. A source of examples is provided by the derivators $\mathrm{HO}(\mathcal{M})$ associated to stable Quillen model categories $\mathcal{M}$.

Recall from [14], §1.19, that given any triangulated derivator $\mathbb{D}$ and small category $X$, the category $\mathbb{D}(X)$ has a canonical triangulated structure. In particular, the category $\mathbb{D}(e)$ is triangulated. Finally, given derivators $\mathbb{D}$ and $\mathbb{D}^{\prime}$, we denote by $\underline{\operatorname{Hom}}\left(\mathbb{D}, \mathbb{D}^{\prime}\right)$ the category of all morphisms of derivators and by $\underline{\operatorname{Hom}}_{!}\left(\mathbb{D}, \mathbb{D}^{\prime}\right)$ the category of morphisms of derivators which preserve arbitrary homotopy colimits; consult [13], $\$ 3.25$, for details.

Acknowledgments. The author is very grateful to Alexander Beilinson, DenisCharles Cisinski, Maxim Kontsevich, Haynes Miller, Carlos Simpson, and Bertrand Toën for stimulating conversations, as well as to the anonymous referee for his comments and corrections, which greatly improved the article. He would also like to thank the Department of Mathematics of MIT for its hospitality and excellent working conditions.

\section{References}

[1] Y. André, Motifs de dimension finie (d'après S.-I. Kimura, P. O'Sullivan, ...). Astérisque 299 (2005), 115-145. Zbl 1080.14010 MR 2167204

[2] Y. André, An introduction to motivic zeta functions of motives. In Motives, quantum field theory, and pseudodifferential operators, Clay Math. Proc. 12, Amer. Math. Soc., Providence, RI, 2010, 3-17. Zbl 1214.81094 MR 2762522

[3] Y. André and B. Kahn, Nilpotence, radicaux et structures monoïdales. Rend. Sem. Mat. Univ. Padova 108 (2002), 107-291. Zbl 1165.18300 MR 1956434

[4] P. Balmer, Spectra, spectra, spectra - tensor triangular spectra versus Zariski spectra of endomorphism rings. Algebr. Geom. Topol. 10 (2010), 1521-1563. Zbl 1204.18005 MR 2661535

[5] P. Balmer and M. Schlichting, Idempotent completion of triangulated categories. J. Algebra 236 (2001), 819-834. Zbl 0977.18009 MR 1813503

[6] A. A. Beilinson, The derived category of coherent sheaves on $\mathbf{P}^{n}$. Selecta Math. Soviet. 3 (1984), 233-237. Zbl 0545.14012 MR 863137 
[7] F. Bittner, The universal Euler characteristic for varieties of characteristic zero. Compos. Math. 140 (2004), 1011-1032. Zbl 1086.14016 MR 2059227

[8] A. J. Blumberg and M. A. Mandell, Localization theorems in topological Hochschild homology and topological cyclic homology. Geom. Topol. 16 (2012), 1053-1120. Zbl 06068621 MR 2928988

[9] A. I. Bondal and M. M. Kapranov, Enhanced triangulated categories. Mat. Sb. 181 (1990), 669-683; ; English transl. Math. USSR-Sb. 70 (1991), 93-107. Zbl 0729.18008 MR 1055981

[10] A. I. Bondal, M. Larsen, and V. A. Lunts, Grothendieck ring of pretriangulated categories. Internat. Math. Res. Notices 2004 (2004), no. 29, 1461-1495. Zbl 1079.18008 MR 2051435

[11] A. Bondal and M. van den Bergh, Generators and representability of functors in commutative and noncommutative geometry. Moscow Math. J. 3 (2003), 1-36. Zbl 1135.18302 MR 1996800

[12] F. Borceux, Handbook of categorical algebra. Vol. 2, Encyclopedia Mathe. Appl. 51, Cambridge University Press, Cambridge 1994. Zbl 0843.18001 MR 1313497

[13] D.-C. Cisinski, Propriétés universelles et extensions de Kan dérivées. Theory Appl. Categ. 20 (2008), No. 17, 605-649. Zbl 1188.18009 MR 2534209

[14] D.-C. Cisinski and A. Neeman, Additivity for derivator K-theory. Adv. Math. 217 (2008), 1381-1475. Zbl 1141.19003 MR 2382732

[15] D.-C. Cisinski and G. Tabuada, Non-connective $K$-theory via universal invariants. Compos. Math. 147 (2011), 1281-1320. Zbl 1247.19001 MR 2822869

[16] D.-C. Cisinski and G. Tabuada, Symmetric monoidal structure on non-commutative motives. J. K-Theory 9 (2012), 201-268. Zbl 1256.19002 MR 2922389

[17] S. del Baño Rollin and V. Navarro Aznar, On the motive of a quotient variety. Collect. Math. 49 (1998), 203-226. Zbl 0929.14033 MR 1677089

[18] P. Deligne, Catégories tensorielles. Moscow Math. J. 2 (2002), 227-248. Zbl 1005.18009 MR 1944506

[19] V. Drinfeld, DG quotients of DG categories. J. Algebra 272 (2004), 643-691. Zbl 1064.18009 MR 2028075

[20] V. Drinfeld, DG categories. University of Chicago geometric Langlands seminar 2002. Notes available at www.math.utexas.edu/users/benzvi/GRASP/lectures/Langlands.html.

[21] H. Gillet and C. Soulé, Descent, motives and K-theory. J. Reine Angew. Math. 478 (1996), 127-176. Zbl 0863.19002 MR 1409056

[22] H. Gillet and C. Soulé, Motivic weight complexes for arithmetic varieties. J. Algebra 322 (2009), 3088-3141. Zbl 1186.14020 MR 2567412

[23] A. Grothendieck, Les Dérivateurs. Manuscript, 1990-91. Available at http://www.math.jussieu.fr/ maltsin/groth/Derivateurs.html

[24] V. Guletski1̆, Finite-dimensional objects in distinguished triangles. J. Number Theory 119 (2006), 99-127. Zbl 1102.14003 MR 2228952

[25] V. Guletskiĭ and C. Pedrini, Finite-dimensional motives and the conjectures of Beilinson and Murre. K-Theory 30 (2003), 243-263. Zbl 1060.19001 MR 2064241 
[26] U. Jannsen, S. Kleiman and J.-P. Serre (eds.), Motives. (Seattle, WA, 1991) Proc. Sympos. Pure Math. 55, part 1 and 2. Amer. Math. Soc., Providence, RI, 1994. Zbl 0788.00054 MR 1265518 MR 1265549

[27] D. Kaledin, Motivic structures in non-commutative geometry. In Proc. Internat. Congr. Mathematicians, Vol. II, Hindustan Book Agency, New Delhi 2010, 461-496. Zbl 1245.14005 MR 2827805

[28] M. Kapranov, The elliptic curve in the S-duality theory and Eisenstein series for KacMoody groups. Preprint, 2000. arXiv:math/0001005

[29] B. Keller, On the cyclic homology of ringed spaces and schemes. Doc. Math. 3 (1998), 231-259. Zbl 0917.19002 MR 1647519

[30] B. Keller, On the cyclic homology of exact categories. J. Pure Appl. Algebra 136 (1999), 1-56. Zbl 0923.19004 MR 1667558

[31] B. Keller, On differential graded categories. In Proc. Internat. Congr. Mathematicians. Vol. II, European Math. Soc. Publ. House, Zürich 2006, 151-190. Zbl 1140.18008 MR 2275593

[32] S.-I. Kimura, Chow groups are finite dimensional, in some sense. Math. Ann. 331 (2005), 173-201. Zbl 1067.14006 MR 2107443

[33] M. Kontsevich, Triangulated categories and geometry. Course at ENS, Paris 1998. Notes available at http://www.math.uchicago.edu/ mitya/langlands.html

[34] M. Kontsevich, Non-commutative motives. Talk at the Institute for Advanced Study on the occasion of the 61st birthday of Pierre Deligne, October 2005. Video available at http://video.ias.edu/Geometry-and-Arithmetic

[35] M. Kontsevich, Notes on motives in finite characteristic. In Algebra, arithmetic, and geometry: in honor of Yu. I. Manin. Vol. II, Progr. Math. 270, Birkhäuser, Boston 2009, 213-247. Zbl 05778256 MR 2641191

[36] M. Kontsevich, Mixed non-commutative motives. Talk at the Workshop on Homological Mirror Symmetry and Related Topics, University of Miami, 2010. Notes available at http://www-math.mit.edu/ auroux/frg/miami10-notes/

[37] E. Looijenga, Motivic measures. Astérisque 276 (2002), 267-297. Zbl 0996.14011 MR 1886763

[38] V. A. Lunts and D. O. Orlov, Uniqueness of enhancement for triangulated categories. $J$. Amer. Math. Soc. 23 (2010), 853-908. Zbl 1197.14014 MR 2629991

[39] Yu. I. Manin, Correspondences, motifs and monoidal transformations. Mat. Sb. (N.S.) 77 (119) (1968), 475-507; English transl. Math. USSR-Sb. 6 (1968), 439-470. Zbl 0199.24803 MR 0258836

[40] C. Mazza, Schur functors and motives. K-Theory 33 (2004), 89-106. Zbl 1071.14026 MR 2131746

[41] D. Quillen, Higher algebraic $K$-theory: I. In Algebraic K-theory I-Higher K-theories (Proc. Conf., Battelle Memorial Inst., Seattle, Wash., 1972), Lecture Notes in Math. 341, Springer, Berlin 1973, 85-147. Zbl 0292.18004 MR 0338129

[42] M. Schlichting, Negative $K$-theory of derived categories. Math. Z. 253 (2006), 97-134. Zbl 1090.19002 MR 2206639 
[43] A. J. Scholl, Classical motives. In Motives (Seattle, WA, 1991), Proc. Sympos. Pure Math. 55, Amer. Math. Soc., Providence, RI, 1994, 163-187. Zbl 0814.14001 MR 1265529

[44] G. Tabuada, Invariants additifs de dg-catégories. Internat. Math. Res. Notices 2005 (2005), 3309-3339. Zbl 1094.18006 MR 2196100

[45] G. Tabuada, Higher $K$-theory via universal invariants. Duke Math. J. 145 (2008), 121-206. Zbl 1166.18007 MR 2451292

[46] G. Tabuada, Generalized spectral categories, topological Hochschild homology and trace maps. Algebr. Geom. Topol. 10 (2010), 137-213. Zbl 1206.55012 MR 2580431

[47] R. W. Thomason and T. Trobaugh, Higher algebraic $K$-theory of schemes and of derived categories. In The Grothendieck Festschrift, Vol. III, Progr. Math. 88, Birkhäuser, Boston 1990, 247-435. Zbl 0731.14001 MR 1106918

Received March 8, 2011; revised July 23, 2011

G. Tabuada, Department of Mathematics, Massachusetts Institute of Technology, Building E17, Cambridge, MA 02139-4307, U.S.A., and Departamento de Matematica e CMA, FCT-UNL, Quinta da Torre, 2829-516 Caparica, Portugal

E-mail: tabuada@math.mit.edu 